\title{
Effect of 9 $\alpha$-Fluorocortisol on the Excretion of Urinary Digoxin-like Substance in Normotensive Men.
}

\author{
The Second Department of Internal Medicine School of \\ Medicine Kanazawa University Kanazawa 920 \\ Fukui Junkanki Hospital, Internal Medicine Fukui 910
}

\author{
Toshio MORISE, Isamu MIYAMORI, Senshu HIFUMI, Shinya OKAMOTO, \\ Masatoshi IKEDA, YoshiYu TAKEDA, Hideo KOSHIDA, \\ SYUICHIRO YASUHARA AND RYOYU TAKEDA
}

\begin{abstract}
In order to investigate the possible role of mineralocorticoid in the regulation of digoxin-like substance (DLS), $9 \alpha$-fluorocortisol (9-F) was administered to 6 healthy men and urinary exeretion of DLS was measured. The administration of $0.6 \mathrm{mg}$ of 9-F caused slight increases in body weight and blood pressure and significant decreases in urinary $\mathrm{Na}$ excretion, plasma renin activity and plasma aldosterone, which indicate the expansion of extracellular fluid (ECF) volume by $9-\mathrm{F}$ administration. Urinary excretion of DLS decreased significantly from the baseline level of $43.3 \pm 2.6$ (SEM) to $29.8 \pm 5.1$ (SEM) $\mathrm{ng}$ /day ; digoxin equiv. after 9-F. These results suggest that a large dose of mineralocorticoid may suppress DLS despite an increase in the ECF volume.
\end{abstract}

The existence of a natriuretic factor in human urine was reported by Clarkson et al., 1976. Since then many efforts have been made to characterize this natriuretic factor. Its inhibitory properties in $\mathrm{Na}-\mathrm{K}$ ATPase activity (Gonic et al., 1977), and more recently its cross-reactivity with antidigoxin antibody (Gruber et al., 1980) appear to represent major progress in the studies on this factor. We have previously demonstrated that natriuretic factors can be measured quantitatively, as digoxinlike substance (DLS) by radioimmunoassay (RIA) using anti-digoxin antibody (Morise et al., 1985). We found that DLS showed a positive correlation with salt intake, sug- gesting a possible role of DSL in sodium homeostasis.

In the meanwhile, it has been shown that an increase in salt intake suppresses the mineralocorticoid secretion through an increase in extracellular fluid volume (ECF). Therefore, it may be argued that a change in mineralocorticoid in addition to that of ECF volume could also be involved in an increase in DLS excretion during high salt intake. In order to further clarify the dual effects of ECF volume and mineralocorticoid on DLS in a high sodium state, we examined the effects of mineralocorticoid on DLS excretion in man by using $9 \alpha$-fluorocortisol (9-F). 


\section{Material and Method}

First we studied whether or not the urinary excretion of DLS reflects the plasma DLS concentration. Twenty-four hour urine was collected from 10:00 a.m. on the first day to 10:00 a.m. on the second day from 19 hospitalized patients with essential hypertension in our clinic at Kanazawa University. Blood was withdrawn into a siliconized glass tube with EDTA at 6:00 a.m. of the second day and centrifuged immediately. Plasma was stored at $-20^{\circ} \mathrm{C}$ until its assay. Urine and plasma DLS were measured after single extraction with a reversed phase cartridge column (Fig. 1) with RIA as previously reported (Morise et al., 1985).

Furthermore, in order to test the interference of steroids including aldosterone and 9-F with the assay system of DLS, their cross-reactivity to anti-digoxin antibody was examined.

After their informed concent was obtained, 6 male volunteers (mean age; 24 years) from the School of medicine, Kanazawa University were studied. These 6 volunteers were prohibited from smoking and drinking alcohol for 2 weeks before the test, and given orally $0.6 \mathrm{mg} /$ day of $9-\mathrm{F}$ once in the morning for three days. Twenty-four hour urine was collected and a blood sample withdrawn at 6:00 a.m. every day, and urinary excretion of DLS, $\mathrm{Na}, \mathrm{K}$, creatinine $(\mathrm{Cr})$, urinary volume, serum $\mathrm{Na}, \mathrm{K}, \mathrm{Cr}$ concentration, plasma renin activity (PRA) and plasma aldosterone were measured. Blood pressure (BP) and body weight (BW) were also measured every day. The electrolytes were determined by flame photometer, and PRA and plasma aldosterone were measured by RIA as previously reported (Takeda et al., 1976). Statistical analyses were made using Student's $t$ test, and 0.05 level of probability was regarded as significant.

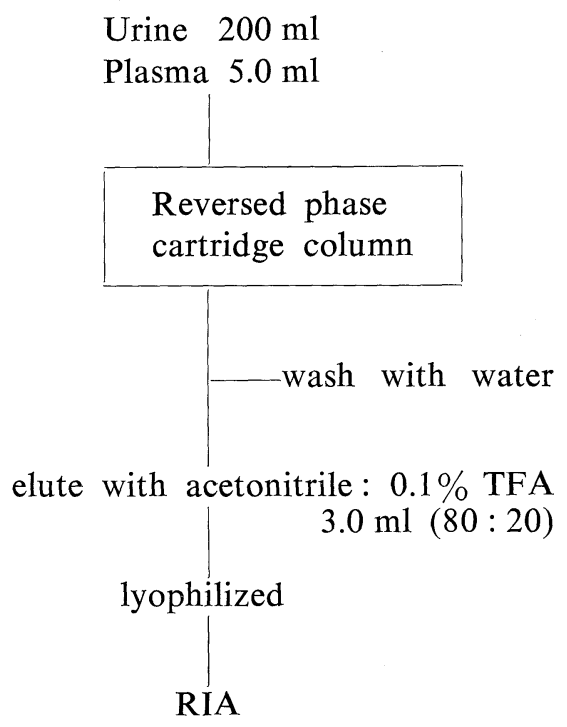

Fig. 1. Extraction procedure for digoxin-like substance.

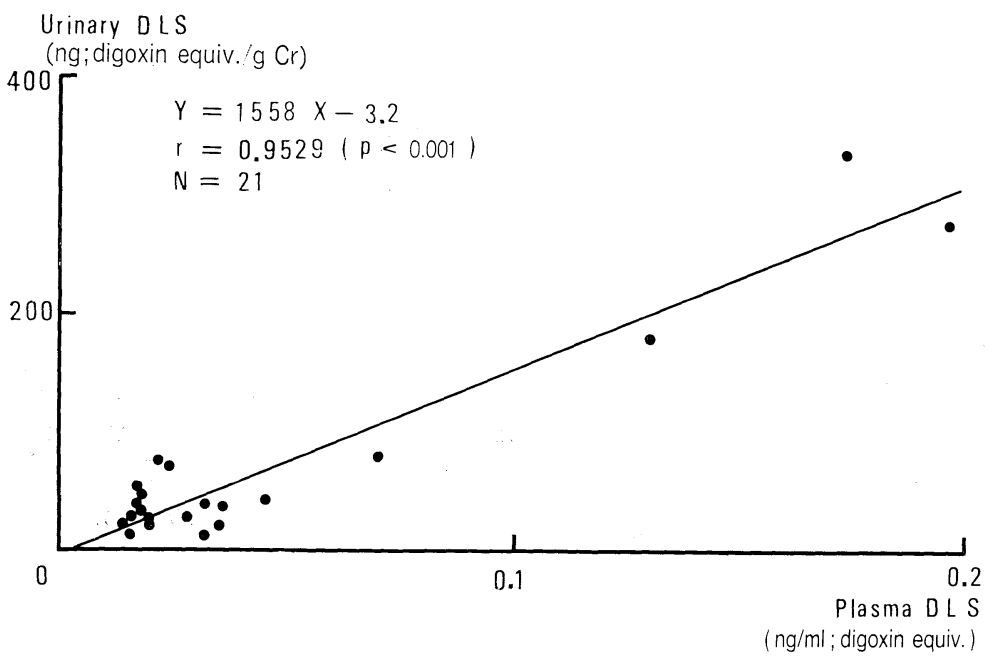

Fig. 2. Correlation between urinary excretion of digoxin-like substance(DLS) and plasma DLS concentration. 
Table 1. Cross-reactivity of steroids with anti-digoxin antibody.

\begin{tabular}{lc}
\hline \hline Steroid & Cross-reactivity \\
\hline d-aldosterone & $<0.0005 \%$ \\
$9 \alpha$-fluorocortisol & $<0.001 \%$ \\
Pregnenolone & $<0.0001 \%$ \\
Progesterone & $<0.001 \%$ \\
Corticosterone & $<0001 \%$ \\
Cortisol & $<0.0005 \%$ \\
$\beta$-cortolone & $<0.0001 \%$ \\
11-dehydrocorticosterone & $<0.001 \%$ \\
11-deoxycortisol & $<0.001 \%$ \\
\hline
\end{tabular}

Table 2. Comparison of clinical characteristics before and after $9 \alpha$-fluorocortisol (9F) administration. Data are shown as mean \pm SEM.

\begin{tabular}{|c|c|c|}
\hline & before $9 \alpha-\mathrm{F}$ & after $9 \alpha-\mathrm{F}$ \\
\hline Body weight $(\mathrm{kg})$ & $61.8 \pm 4.6$ & $63.2 \pm 4.9$ \\
\hline $\begin{array}{l}\text { Mean blood } \\
\text { pressure }(\mathrm{mmHg})\end{array}$ & $92.3 \pm 3.3$ & $93.8 \pm 5.6$ \\
\hline Ht. $\quad(\%)$ & $40.8 \pm 2.0$ & $37.0 \pm 1.0^{*}$ \\
\hline PRA $\quad(\mathrm{ng} / \mathrm{ml} / \mathrm{h})$ & $1.3 \pm 0.3$ & $0.1 \pm 0.1^{*}$ \\
\hline P-aldo（ng/dl) & $5.6 \pm 3.1$ & $2.6 \pm 0.3^{*}$ \\
\hline $\mathrm{S}-\mathrm{Na} \quad(\mathrm{mEq} / \mathrm{l})$ & $138.0 \pm 1.1$ & $138.0 \pm 0.4$ \\
\hline$(\mathrm{mEq} / 1)$ & $4.0 \pm 0.2$ & $3.2 \pm 0.1^{*}$ \\
\hline$(\mathrm{mEq} / \mathrm{D})$ & $249.6 \pm 18.8$ & $190.8 \pm 36.9^{*}$ \\
\hline$(\mathrm{mEq} / \mathrm{D})$ & 41.8 上 11.0 & $63.6 \pm 18.6$ \\
\hline
\end{tabular}

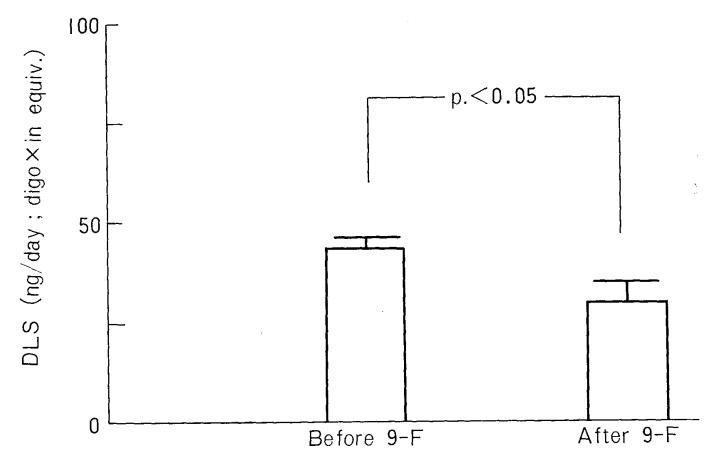

Fig. 3. Urinary digoxin-like substance (DLS) before and after $9 \alpha$-fluorocortisol (9-F) administration.

\section{Results}

There was a significant positive correration $(\mathrm{p}<0.001)$ between the plasma DLS concentration and urinary excretion of DLS (Fig. 2), and it was confirmed that urinary excretion of DLS well reflected the plasma concentration. Moreover, cross reactivity of various steroids to anti-digoxin antibody was less than $0.001 \%$ (Table 1.) and it was considered that they did not interfere with the DLS RIA.

After the administration of 9-F, BW and BP slightly increased (not statistically significant), and urinary sodium excretion, plasma aldosterone and PRA decreased significantly $(\mathrm{p}<0.05$, Table 2.). Urinary excretion of DLS decreased significantly $(\mathrm{p}<0.05)$ from $43.3 \pm 2.6$ before $9-\mathrm{F}$ administration to $29.8 \pm$ $5.1 \mathrm{ng} /$ day; digoxin equiv. after 9-F (Fig. 3).

\section{Discussion}

Recent evidence indicates that substance(s) which cross-reacts significantly with anti-digoxin antibody exist in human blood and urine (Gruber et al., 1980, Klingmuller et al., 1982). Further characterization of this substance disclosed its inhibition of Na-K-ATPase activity (Gonic et al., 1977), and this substance was indentified as an endogenous natriuretic factor by various bioassay techniques (Klingmuller et al., 1982). We measured DLS by RIA using anti-digoxin antibody, and demonstrated that urinary DLS excretion increased when salt intake increased and that there was a positive correlation between them (Morise et al., 1985). However, when salt intake increases, an increase in ECF volume and suppression of mineralocorticoid should accompany it. Therefore, it is likely that mineralocorticoid plays a role in the regulation of DLS. In order to investigate the relationship between mineralocorticoid and DLS, we orally ad- 
ministered 9-F, one of the mineralocorticoids, to men and studied its effect on the urinary excretion of DLS. 9-F is a synthetic steroid which has some glucocorticoid activity but also has a very strong mineralocorticoid activity. The dose of $0.6 \mathrm{mg} /$ day employed in the present study is sufficient to cause excessive hypermineralocorticoidism in man (Takeda et al., 1985). In the present study, it is suggested that the ECF volume was expanded due to mineralocorticoid excess by the results such as BW gain, elevation of BP, suppression of plasma aldosterone and PRA. Nevertheless, urinary DLS decreased significantly $(p<0.05)$ in all cases. The present results suggest that $9-\mathrm{F}$ may suppress the DLS excretion. This decrease in DLS with 9-F treatment does not conflict with the results reported by Hegyzary in 1977 showing that a high dose of aldosterone has been known to enhance myocardial ATPase activity in guinea pigs.

Our present results indicating that DLS excretion is stimulated when ECF volume is expanded by high salt diet, while it is suppressed when excessive mineralocorticoid is administered, correspond with those experimental results in DOCA-salt hypertensive rat reported by Stern et al., 1984. In DOCAhypertensive rats, controversial results were reported on Na-K-ATPase activity (Friedman et al., 1976, Haddy et al., 1981). Regarding the Na-K-ATPase activity in DOCA-hypertensive rats, Friedman et al. reported an increase in enzymatic activity, while Haddy et al., reported a decrease therein. This controversy may result from the complicated relationship between two factors, that is, increased ECF volume and excessive mineralocorticoid in DOCA-salt hypertensive rats. Stern et al., 1984 skilfully combined experimental conditions such as high salt diet, DOCA treatment, adrenalectomy and so on and demonstrated that the group which received uninephrectomy and saline loading showed a decrease in Na-K-ATPase activity, and the group which was given DOCA in addition to uninephrectomy and saline loading showed no significant decrease therein compared with the control group. From these results they suggested that increased ECF volume might decrease Na-K-ATPase activity of kidney and mineralocorticoid might increase Na-K-ATPase activity, They suggested that a certain circulating Na-KATPase inhibitor may be responsible for their results but they made no measurements. Taking our experimental results into consideration, it is suggested that DLS, a circulating Na-K-ATP-ase inhibitor, may play a role in the regulation of Na-K-ATPase activity in DOCAsalt hypertensive rats.

In our study of human subjects, urinary DLS excretion was $37.5 \pm 5.5 \mathrm{ng} /$ day for the normal control $(\mathrm{n}=27), 102.5 \pm 18.1 \mathrm{ng} / \mathrm{day}$ for patients with essential hypertension $(\mathrm{EHT}, \mathrm{n}=23), 56.8 \pm 17.3 \mathrm{ng} /$ day for patients with primary aldosteronism $(\mathrm{PA}, \mathrm{n}=4)$ and $247.7 \pm 84.5 \mathrm{ng} /$ day for patients with chronic heart failure $(\mathrm{CHF}, \mathrm{n}=11)$, respectively (unpublished data, presented to the 5th Conference on Sodium and Hypertension, Tokyo, 1985). PA, which is a disease responsible for sodium retention similar to CHF, presumably shows a high level of urinary DLS. However, our measurements revealed that PA showed far lower values than CHF, with a much lower value than EHT. Although we were very puzzled as to how to interpret these results, the fact that PA did not show a very high urinary DLS excretion level can be explained by assuming that sodium retention and a subsequent increase in ECF stimulate DLS excretion and mineralocorticoid excess suppresses its excretion.

Whether or not the above hypothesis is correct can only be ascertained after further studies including a search of a chemical structure of DLS and the site of its production. 


\section{Acknowledgements}

This work was supported in part by a grant from the Ministry of Health and Welfare "Disorders of Steroid Hormones" Research Committee, Japan, 1985.

\section{References}

Clarkson, E. M., M. R. Sheelagh and H. E. de Wardener (1976). Two natriuretic substances in extracts of urine from normal man when salt-depleted and salt-loaded. Kidney. Int. 10, 381-394.

Friedman, S. M. and C. L. Friedman (1976). Cell permeability, sodium transport and hypertensive process in the rat. Circ. Res. 39, 433441.

Gonic, H. C., H. J. Kramer, W. Paul and E. Lu (1977). Circulating inhibitor of sodium-potassium-activated adenosine triphosphatase after expansion of extracellular fluid volume in rats. Clin. Sci. Mol. Med. 53, 329-334.

Gruber, K. A., J. M. Whitaker and V. M. Buckalew Jr (1980). Endogenous digitalis-like substance in plasma of volume-expanded dogs. Nature. 287, 743-745.
Haddy, F., M. Pammani, D. Clough and S. Hunt (1981). Role of a humoral sodium-potassium pump inhibitor in experimental low renin hypertension. Life Sci. 30, 571-575.

Hegyzary C (1977). Effect of aldosterone and methylprednisolone on cardiac Na-K-ATPase. Experientia. 33, 1280-1982.

Klingmuller, D. E., E. Weiler and H. J. Kramer (1982). Digoxin-like natriuretic activity in the urine of salt loaded healthy subjects Klin. Wochenschr. 60, 1249-1253.

Morise, T., I. Miyamori, S. Hifumi, S. Okamoto, M. Ikeda, Y. Takeda, H. Koshida, S. Yasuhara and R. Takeda (1985). Effect of sodium intake on the excretion of urinary natriuretic factor in essential hypertensives. Endocrinol. Japon. 32, 91-97.

Stern, N., W.J. B. Frances, N. Vlachakis, P. Eggena and R. Sowers (1984). Sodium-potassium ATPase in deoxycorticosterone-salt hypertension: Opposing effects of sodium load and mineralocorticoids. Endocrinology 114, 624-628.

Takeda, R., S. Morimoto, K. Utida and I. Miyamori (1976). Changes in plasma renin activity and plasma aldosterone in the induced paralytic attack of thyrotoxic periodic paralysis. Acta. Endocroinol. $(k b h), 82,715-727$.

Takeda R (1985). Review on internal medicine, 1984. Electrolyte disturbance. II-2, Mineralocorticoids and potassium imbalance. J. Japan. Soc. Int. Med. 74, 702-707. 